Check for updates

Montreal

Cite this as: BMJ 2021;375:n3104 http://dx.doi.org/10.1136/bmi.n3104 Published: 16 December 2021

\title{
Covid-19: Omicron is causing more infections but fewer hospital admissions than delta, South African data show
}

Owen Dyer

Data from South Africa's largest private health insurer suggest that omicron is spreading faster than any previous coronavirus variant and showing signs of immune escape, with both vaccinated and previously infected people more at risk than in previous waves.

More than $90 \%$ of newly sequenced infections in South Africa now involve the omicron variant, and as it displaced delta, vaccine effectiveness dipped, revealed data presented by the insurer Discovery Health at a 14 December briefing. ${ }^{1}$

Two doses of the Pfizer vaccine provided only $33 \%$ protection against infection in this omicron fuelled wave, found the analysis, down from $80 \%$ in South Africa's last, delta fuelled wave. The protection against severe symptoms requiring hospital admission has stood up better, at 70\% in the current wave compared with $93 \%$ in the last.

Omicron also appears more capable than delta of penetrating the immune defences of the previously infected. In South Africa's second (beta variant) wave, new infections were $60 \%$ as likely among the previously infected as they were among the never infected. In the third (delta) wave, the previously infected ran only $40 \%$ as much risk as the never infected. But in the current wave, new cases are $73 \%$ as common among the previously infected as among the never infected.

Discovery Health's data cover the period from 15 November to 7 December and include over 211000 covid test results with their subsequent clinical course. Of these, around 78 ooo were deemed to be omicron infections. About $41 \%$ of those tested had received two doses of Pfizer vaccine. The analysis was conducted in collaboration with the South African Medical Research Council.

\section{Hospital admissions}

The data suggest that infections in the current wave are less likely to lead to hospital admission than in South Africa's previous surges. After adjusting for vaccination status, the risk of hospital admission for newly diagnosed adults is $29 \%$ lower than in the first wave, said Shirley Collie, a statistician at Discovery Health, presenting the findings. "Furthermore," she said, "adults admitted to hospital currently have a lower propensity to be admitted to high care and intensive care units, relative to prior waves."

Among children, conversely, the rate of hospital admission is up $20 \%$. But these cases are mostly mild and the absolute risk in children remains low, said Collie.

Discovery's chief executive Ryan Noach said, "We are hopeful that the current experience of covid-19 caused by the omicron variant-mild disease for the most part-will remain unchanged. Notwithstanding this, we remain concerned that health systems could still come under pressure considering the high rate of spread."

The World Health Organization's director for Africa, Matshidiso Moeti, also described a wave characterised by faster spread and less severe illness. "We are cautiously optimistic," she said at a 14 December briefing, "as we are seeing fewer deaths during the early weeks of this current wave when compared with previous surges."

Researchers from the University of Hong Kong who studied omicron in ex vivo cultures of the respiratory tract found that it replicates 70 times faster than delta in human bronchus, but 10 times slower than delta in human lung tissue. The former finding could explain omicron's rapid spread, the authors wrote, while the latter finding "may be an indicator of lower disease severity." 2

The researchers' data has not yet been published or peer reviewed. Michael Chan Chi-wai, who led the study, cautioned that "by infecting many more people, a very infectious virus may cause more severe disease and death even though the virus itself may be less pathogenic." Given its ability to partially escape immunity from vaccines and past infection, he said, "the overall threat from the omicron variant is likely to be very significant."

Analysis of omicron outbreak based on 211000 covid-19 test results in South Africa. Discovery Health. 14 December 2021. www.discovery.co.za/corporate/news-room

2 HKUMed finds Omicron SARS-CoV-2 can infect faster and better than delta in human bronchus but with less severe infection in lung. 15 December 2021. www.med.hku.hk/en/news/press/20211215-omicron-sars-cov-2-infection.

This article is made freely available for use in accordance with BMJ's website terms and conditions for the duration of the covid-19 pandemic or until otherwise determined by BMJ. You may use, download and print the article for any lawful, non-commercial purpose (including text and data mining) provided that all copyright notices and trade marks are retained. 